\title{
COVID 19: Re-evaluation of Interventional Radiology
}

\author{
Raman Uberoi $^{1} \cdot$ Klaus Hausegger $^{2}$
}

Published online: 20 January 2021

(C) Springer Science+Business Media, LLC, part of Springer Nature and the Cardiovascular and Interventional Radiological Society of Europe (CIRSE) 2021

2020 will go down as one of the worst in memorable history of the human race. It is more than a year since the COVID virus exploded onto the world scene and created one of the greatest human pandemics in history. The COVID global pandemic has had a devastating impact on the economies and healthcare systems across the world. It has highlighted the unpreparedness of virtually every healthcare system in the world, although there have been many previous warnings. SARS in 2003 was a warning shot. More by chance than by design the virus mutated to a more benign form and the epidemic fizzled out, but the world failed to heed the warnings and act decisively to prepare. With COVID we were developing systems and pathways for the first time in the heat of the pandemic when we should already have had these ready and in place. Personal protections devices were not available, and there was a mass scramble around the world to get as many for their healthcare systems as possible.

There have been many publications including in our own journal, and there are many learning points specific to COVID, the high bleeding and thrombo-embolic complications, lung injury and barotrauma. But there are also more generic important working practices to ensure staff and patient safety, such as segregation of patients with different risk by place and time, segregation of staff teams to minimize cross infections, reducing patient movement by undertaking procedures where possible with ultrasound by the bedside, prioritization of patient so urgent procedures continue, and optimization of workflows through rehearsals particularly for our angiographic suites.

Patients can present with minimal symptoms but then rapidly progress to the extremes of acute respiratory distress, multi-organ failure, coagulopathy, thromboembolic disease and disseminated intravascular coagulation [1-4]. Not surprisingly, therefore, intensive care physicians and pulmonologists have been at the forefront in the medical management of these complex and difficult patients. However, the COVID 19 crisis has also highlighted the crucial role that radiology and, in particular interventional radiology, can play in helping to manage many patients with both suspected and positive COVID disease when other surgical treatments have to be restricted or deferred, often because of limited ward and intensive treatment care (ITU) bed capacity. From major COVID centres, there have been multiple reports of the value and importance of IR in treating these complex patients using minimally invasive techniques [5-7]. In addition, some semielective and urgent treatments such as tumor ablations, fistuloplasties, lines and port insertions, angioplasty and stenting for critical limb ischemia have continued, often as day cases procedures, minimizing risk to patients and other hospital staff. IR's have also continued to deliver emergency IR treatments for sepsis, bowel obstruction and hemorrhage control, often putting themselves at significantly increased personal risk.

Raman Uberoi

Raman.Uberoi@ouh.nhs.uk

1 Department of Radiology, John Radcliffe Hospital, Oxford University Hospitals NHS Trust, Oxford, UK

2 Department of Diagnostic and Interventional Radiology, Klagenfurt State Hospital, Klagenfurt, Austria 
This has highlighted the clear divergence which has emerged from the working practices of our colleagues who only undertake imaging radiology. Many units quite rightly have been operating with minimal onsite staff to limit exposure to the COVID pathogen. This has meant that many colleagues who focus on imaging radiology are working either from home or if onsite in protected, restricted environments with no patients and often minimal staff contact, whereas for IR teams it is absolutely necessary to have close direct patient and staff contact as well as being available 24/7 onsite to deliver lifesaving care. Often undertaking procedures in sites around the hospital including the wards and ITUs in very difficult circumstances and limited support. Having appropriate personal protection equipment (PPE) is vitally important to safeguard all staff; however this brings in the added complexities of donning and doffing of PPE all of which can be very difficult to work with, making even the simplest procedures technically more challenging, much longer and uncomfortable. Remaining focused and delivering the best possible treatment in these circumstances can be virtually impossible. However, time and again IR's have demonstrated their skill and resolve in helping these patients, despite the difficulties and personal risks involved.

We all hope that COVID 19 will be a once in a generation occurrence and neither we, or our families will not see this again. Governments have many distractions and short attention spans. Although this pandemic is not yet finished, we have to learn from it and as healthcare professionals we have a responsibility to ensure that we highlight the needs of our patients to our governments and ensure we are ready for the next. No healthcare system was well prepared and we need to learn from this terrible crisis so that we are ready and can act more quickly and decisively in the future. There are, of course, some positives and we must applaud the fantastic and rapid collaboration of healthcare professionals around the world to share knowledge, experience and not least in developing an effective vaccine. There is also the realization of many of the healthcare systems around the world of the central role of radiology, in particular IR in managing and treating patients in these difficult circumstances.

Funding None.

\section{Compliance with Ethical Standards}

Conflict of interest The authors declare that they have no conflict of interest.

Consent for Publication For this type of study consent for publication is not required.

\section{References}

1. Tang N, Bai H, Chen X, Gong J, Li D, Sun Z, Anticoagulant treatment is associated with decreased mortality in severe coronavirus disease, patients with coagulopathy. J Thromb Haemost. 2019;2020. https://doi.org/10.1111/jth.14817.

2. Diaz R, Heller D. Barotrauma And Mechanical Ventilation. StatPearls Publishing; 2020. http://www.ncbi.nlm.nih.gov/pubmed/31424810. Accessed April 19, 2020.

3. Han H, Yang L, Liu R, et al. Prominent changes in blood coagulation of patients with SARS-CoV-2 infection. Clin Chem Lab Med. 2020. https://doi.org/10.1515/cclm-2020-0188.

4. Zhou F, Yu T, Du R, et al. Clinical course and risk factors for mortality of adult inpatients with COVID-19 in Wuhan, China: a retrospective cohort study. Lancet. 2020;395(10229):1054-62. https://doi.org/10.1016/S0140-6736(20)30566-3.

5. Yang Shen, Ying Cui Ning Li, Chen Tian, et al. Emergency responses to Covid-19 outbreak: experiences and lessons from a general hospital in Nanjing, China. Cardiovasc Intervent Radiol (2020) 43:810-819

6. Da Zhuang K. Bien Soo Tan, Ban Hock Tan, Chow Wei Too, Kiang Hiong Tay. Old threat, new enemy: is your interventional radiology service ready for the Coronavirus Disease 2019? Cardiovasc Intervent Radiol. 2020;43:665-6.

7. Jim Zhong, Anubhav Datta,Thomas Gordon, et al. The Impact of COVID-19 on Interventional Radiology Services in the UK Cardiovasc Intervent Radiol (2021) 44:134-140

Publisher's Note Springer Nature remains neutral with regard to jurisdictional claims in published maps and institutional affiliations. 